\title{
NOUVELLe
}

\section{Adaptation de la synthèse protéique du VEGF-C par les cellules tumorales en condition hypoxique}

Florent Morfoisse ${ }^{1,2}$, Edith Renaud ${ }^{2,3}$, Fransky Hantelys ${ }^{2,3}$, Anne-Catherine Prats ${ }^{2,3}$, Barbara Garmy-Susini ${ }^{1,2}$

\author{
${ }^{1}$ Inserm U1037 ; F-31432 Toulouse, France ; \\ 2 université de Toulouse ; \\ ${ }^{3}$ université Paul Sabatier ; Centre de recherches sur le cancer, \\ Toulouse ; TRADGENE; EA4554; F-31432 Toulouse, France. \\ barbara-garmy-susini@inserm.fr
}

> Le système lymphatique a pour rôle principal de drainer les fluides tissulaires et de participer à la surveillance immune. II possède un mur vasculaire beaucoup plus perméable que celui du réseau sanguin, ce qui en fait une voie privilégiée pour la dissémination métastatique [1]. De récentes études ont démontré l'importance de cibler le système lymphatique afin de réduire le processus métastatique [1]. Le système lymphatique a été très peu étudié du fait de la difficulté de le différencier du réseau sanguin jusqu'à ce que de récents travaux identifient des marqueurs spécifiques tels que Prox-1 [2], Podoplanine [3] et Lyve-1 $[4,5](\rightarrow)$. Grâce à ces nouveaux outils, l'apparition de la lymphangio-

$(\rightarrow)$ Voir $\mathrm{m} / \mathrm{s} 2008$, $n^{\circ}$ 6-7, p. 567

genèse, croissance de nouveaux vaisseaux lymphatiques à partir de vaisseaux préexistants, est maintenant clairement établie aussi bien sur le pourtour de la tumeur primaire (Figure 1) que dans les ganglions lymphatiques sentinelles [6].

Deux facteurs de croissance sont spécifiques de la cellule endothéliale lymphatique: les vascular endothelial growth factors-C et -D (VEGF-C et VEGF-D). II a été montré que l'augmentation de l'expression de ces deux facteurs lors du développement des tumeurs solides est directement corrélée à l'apparition de métastases, et donc à un mauvais pronostic [7].

Dans un contexte tumoral, l'hypoxie est le principal stress induisant la formation de nouveaux vaisseaux sanguins [8]. Cependant, le rôle de l'hypoxie dans la formation de vaisseaux lymphatiques reste à explorer. Les vaisseaux lymphatiques sont le plus souvent situés dans des zones hypoxiques de la tumeur et sont eux-mêmes en hypoxie permanente du fait de l'absence d'hématies dans la lymphe. Jusqu'à une date récente, seules quelques études suggéraient un lien entre l'hypoxie et la lymphangiogenèse sans qu'aucun mécanisme moléculaire direct n'ait encore été mis en évidence.

\section{Le stress hypoxique inhibe}

la traduction dépendante de la coiffe Dans les tumeurs, l'hypoxie induit une rapide inhibition globale de la synthèse protéique [9]. Ceci est lié à un blocage du mécanisme classique d'initiation de la traduction dépendante de la coiffe après inhibition de l'activité kinase de mTOR [9]. On observe alors une surexpression et une hypophosphorylation de la protéine $4 \varepsilon-B P$ (elF4E-binding protein), qui va séquestrer le facteur de liaison à la coiffe elF4E, aboutissant à une inhibition de l'initiation de la traduction [10]. Dans ce contexte, la traduction de certains messagers spécifiques ne peut se produire que grâce à des mécanismes alternatifs. Un des principaux mécanismes traductionnels intervenant en cas de stress implique les IRES (internal ribosome entry sites), éléments structuraux présents dans la région 5' non traduite (5'UTR) de certains ARNm, qui leur permettent d'être traduits lorsque la traduction dépendante de la coiffe est bloquée. La plupart des structures IRES sont retrouvées sur les ARNm des protooncogènes et des facteurs de croissance tels que FGF-2, VEGF-A [11].

\section{L'activité IRES du VEGF-C permet} le maintien de la traduction du VEGF-C en condition hypoxique

Nous avons montré, par l'analyse histologique de prélèvements réalisés dans une cohorte de patients atteints de cancer du sein, que le VEGF-C continue à être surexprimé dans les tumeurs, malgré une diminution de la synthèse protéique globale (surexpression de 4E-BPl) [9]. Nous avons déterminé la présence d'une activité IRES sur l'ARNm codant pour le VEGF-C, qui permet sa synthèse tandis que la traduction dépendante de la coiffe est bloquée. Nos résultats montrent que le stress hypoxique réduit la transcription et la traduction dépendante de la coiffe du VEGF-C dans des lignées tumorales in vitro et in vivo en surexprimant et hypophosphorylant $4 \varepsilon-B P 1$ (Figure 2). En parallèle, nous avons démontré que la traduction de I'ARNm du VEGF-C est maintenue en condition hypoxique grâce à une structure IRES située dans la région 5'UTR de l'ARNm. De façon intéressante,

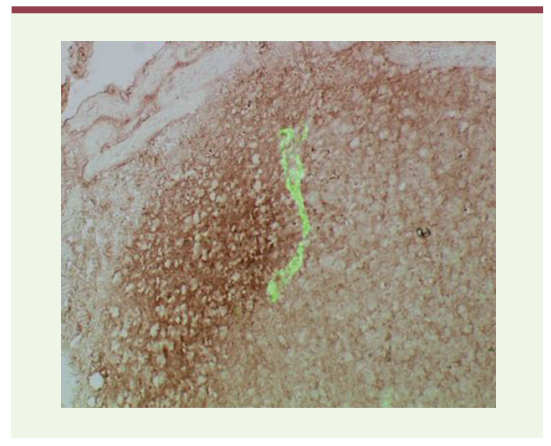

Figure 1. Lyve-1/Pimonidazole. Immunodétection des vaisseaux lymphatiques (Lyve-1) (en vert) en périphérie des zones d'hypoxie (Pimonidazole) dans les tumeurs capan-l. 
l'analyse par SHAPE (selective 2'-hydroxyl acylation analyzed by primer extension) de la structure de ce 5'UTR révèle, malgré une faible conservation de séquence, des structures conservées entre les espèces (souris/ humain), susceptibles d'être les éléments requis pour l'activité de l'IRES. Cette étude montre aussi pour la première fois la stimulation d'une activité IRES au cours du développement tumoral in vivo [9].

\section{L'environnement hypoxique conditionne l'activité IRES}

De manière intéressante, l'analyse de l'activité IRES de l'ARNm du VEGF-C dans les différents compartiments que traverse la cellule tumorale au cours du processus métastatique indique qu'elle est d'autant plus importante que la cellule se trouve dans un système lymphatique hypoxique ( 5 à 18 fois plus de traduction dépendante de I'IRES dans le ganglion lymphatique drainant que dans la tumeur primaire). Afin de s'affranchir de la composante hypoxique du microenvironnement, nous avons étudié l'activité IRES chez des souris invalidées pour la prolyl hydroxylase $2\left(\mathrm{PHD2}^{+/-}\right)$. Chez ces souris, HIFl (hypoxia induced factor 1) est stabilisé, et on assiste à une normalisation des vaisseaux et une meilleure oxygénation des tumeurs. On observe alors une diminution de l'activité IRES de I'ARNm codant pour le VEGF-C alors que le taux de protéines est maintenu, indiquant une reprogrammation de l'expression génique du VEGF-C vers une traduction dépendante de la coiffe lorsque les taux d'oxygène augmentent. Nous avons également mis en évidence que la traduction dépendante de l'IRES de I'ARNm VEGF-C est indépendante de HIF-l $\alpha$.

$\varepsilon$ n conclusion, nous avons identifié une nouvelle régulation de la synthèse du VEGF-C dans des conditions hypoxiques, à la fois dans les tumeurs primaires et dans les ganglions lymphatiques métastatiques. Contrairement à ce qui se produit pour la plupart des cibles de I'hypoxie, qui sont induites au niveau transcriptionnel par HIF, le VEGF-C est induit par un mécanisme dépendant de

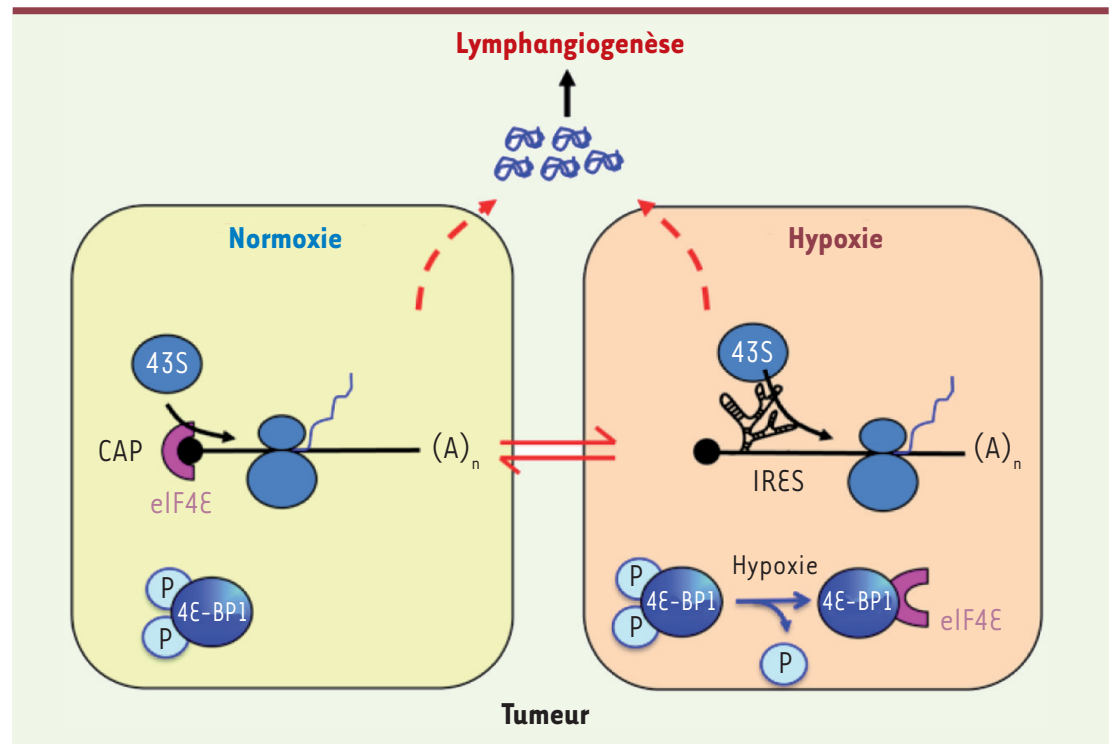

Figure 2. Mécanismes de traduction en condition d'hypoxie : le rôle d'un IRES. En condition normoxique, le complexe d'initiation de la traduction est recruté au niveau de la coiffe de l'ARNm. En présence d'un stress hypoxique, $4 \varepsilon-B P 1$ est déphosphorylée et séquestre elF4E dans le cytoplasme. La traduction globale est alors inhibée excepté pour une faible quantité d'ARNm comportant, dans la région 5' non traduite, un site d'entrée interne du ribosome (IRES) qui permet le recrutement direct de la petite sous-unité du ribosome.

I'IRES, totalement post-transcriptionnel (Figure 2). Cette régulation moléculaire se distingue donc du mécanisme classiquement décrit pour la stimulation de l'angiogenèse par l'hypoxie [12, 13].

\section{Conclusion}

En conclusion, notre étude participe activement à la compréhension des mécanismes développés par les cellules tumorales pour assurer leur dissémination par voie lymphatique dans des conditions pauvres en oxygène. Les vaisseaux lymphatiques sont sévèrement hypoxiques, non seulement parce qu'ils ne transportent pas d'hématies, mais également parce qu'ils résident dans des régions très hypoxiques au sein des tissus et des tumeurs. Un tel environnement hypoxique supprime la traduction dépendante de la coiffe de l'ARNm $\mathrm{du}$ VEGF-C dans les cellules tumorales lorsqu'elles parcourent le système lymphatique en direction des ganglions, rendant inefficaces les inhibiteurs de la traduction tels que les inhibiteurs de mTOR. Cependant, grâce au démarrage de la traduction de I'ARNm du
VEGF-C dépendante de I'IRES, les celIules tumorales peuvent maintenir, voire augmenter, la production de ce facteur lymphangiogénique clé en conditions hypoxiques, et assurer ainsi une augmentation de la croissance des vaisseaux lymphatiques par l'intermédiaire desquels elles essaiment pour former des métastases. Ainsi, notre étude aura mis en lumière la régulation traductionnelle dépendante d'un IRES comme un mécanisme clé permettant aux cellules tumorales de s'adapter au stress hypoxique afin de stimuler la lymphangiogenèse et de former des métastases dans les ganglions lymphatiques. $\diamond$ Lymphangiogenic gene expression adaptation in tumor hypoxic environment

\section{LIENS D'INTÉRÊT}

Les auteurs déclarent n'avoir aucun lien d'intérêt concernant les données publiées dans cet article.

\section{RÉFÉRENCES}

1. Alitalo, K. The lymphatic vasculature in disease. Nat Med 2011; 17 : 1371-80.

2. Wigle JT, Oliver G. Proxl function is required for the development of the murine lymphatic system. Cell $1999 ; 98: 769-78$. 


\section{RÉFÉRENCES}

3. Breiteneder-Geleff S, Soleiman A, Horvat R, et al. Podoplanin: a specific marker for lymphatic endothelium expressed in angiosarcoma. Verh Dtsch Ges Pathol $1999 ; 83: 270-5$.

4. Banerji S, Ni J, Wang SX, et al. LyVE-1, a new homologue of the CD44 glycoprotein, is a lymphspecific receptor for hyaluronan. J Cell Biol 1999 $144: 789-801$.

5. Jaffredo T. Origine veineuse des vaisseaux lymphatiques chez les mammifères: I'hypothèse de Sabin vérifiée. Med Sci (Paris) 2008 ; 24 : 567-9.
6. Garmy-Susini B, Avraamides CJ, Desgrosellier JS, et al. PI3Kalpha activates integrin alpha4betal to establish a metastatic niche in lymph nodes. Proc Natl Acad Sci USA 2013; 110 : 9042-7.

7. Alitalo A, Detmar M. Interaction of tumor cells and lymphatic vessels in cancer progression. Oncogene 2012 ; 31 : 4499-508.

8. Mazzone M, Dettori D, Leite de Oliveira R, et al. Heterozygous deficiency of PHD2 restores tumor oxygenation and inhibits metastasis via endothelial normalization. Cell 2009 ; $136: 839-51$.

9. Braunstein S, Karpisheva K, Pola C, et al. A hypoxiacontrolled cap-dependent to cap-independent translation switch in breast cancer. Mol Cell 2007 ; 28 : 501-12.
10. Morfoisse F, Kuchnio A, Frainay C, et al. Hypoxia induces VEGF-C expression in metastatic tumor cells via a HIF-lalpha-independent translation-mediated mechanism. Cell Rep $2014 ; 6$ : 155-67.

11. Conte C, Riant $\varepsilon$, Toutain C, et al. FGF2 translationally induced by hypoxia is involved in negative and positive feedback loops with HIF-lalpha. PLoS One $2008 ; 3$ : e3078.

12. Hasmim M, Messai Y, Noman MZ, Chouaib S. L'hypoxie tumorale: un déterminant clé de la réactivité stromale et de la réponse anti-tumorale tumor responses. Med Sci (Paris) $2014 ; 30: 422-8$.

13. Azzi $S$, Gavard J. Vaisseaux sanguins et tumeurs ou l'art du dialogue. Med Sci (Paris) 2014 ; 30 : 408-14.

\section{NOUVELle}

Les anticorps anti-VIH-1
et la transmission virale
de cellule à cellule

Marine Malbec ${ }^{1,2}$, Hugo Mouquet ${ }^{2}$, Olivier Schwartz ${ }^{1}$
$>$ Une des avancées majeures de ces cinq dernières années concernant la lutte contre l'infection par le virus de l'immunodéficience humaine de type 1 ( $\mathrm{VIH}-\mathrm{l}$ ) est l'isolement et la caractérisation d'anticorps humains neutralisants, à large spectre ou broadly neutralizing antibodies (bNAb), agissant à basse concentration sur la majorité des isolats viraux

[11] $(\rightarrow)$. L'activité antivirale de ces anticorps était générale-

$\rightarrow$ Voir la Synthèse de M. Peressin et al., $m / s n^{\circ} 1$, janvier 2014, page 69

ment mesurée in vitro contre des particules virales libres. Cependant, le VIH-I se propage préférentiellement et beaucoup plus efficacement par contacts directs entre cellules. Nous avons donc étudié la capacité de ces anticorps à inhiber la transmission virale de cellule à cellule. Seuls quelques bNAb présentent cette propriété, et bloquent chacune des étapes de l'infection intercellulaire. Des essais cliniques avec ces anticorps devraient permettre d'évaluer leur potentiel thérapeutique.

\section{Les anticorps à large spectre neutralisant le VIH-1}

Un mécanisme de défense du système immunitaire en réponse aux infections virales est la production d'anticorps neutralisants. Trente ans après la découverte du VIH-1, un vaccin induisant de tels anticorps se fait toujours attendre. Cela est en partie dû à la grande variabilité génétique du virus. Le VIH-l est divisé en groupes (ou clades), eux-mêmes subdivisés en sous-types parmi lesquels on trouve différentes souches. Certains patients, après deux à quatre ans d'infection, développent des anticorps neutralisants à large spectre (bNAb), dits cross-clade, neutralisant in vitro la plupart des isolats de VIH-l. Alors qu'ils étaient considérés comme des exceptions il y a quelques années, ces individus représenteraient jusqu'à 10 à $20 \%$ des personnes infectées. Malheureusement pour ces patients, la présence de bNAb n'est pas corrélée avec une évolution favorable de la maladie en absence de traitement antirétroviral, des virus résistants à la réponse humorale apparaissant continuellement [1]. Jusqu'à récemment, les anticorps neutralisants clonés à partir de lymphocytes $B$ humains disponibles étaient souvent peu actifs ou peu cross-réactifs. La mise au point de nouvelles techniques
${ }^{1}$ Institut Pasteur, département de virologie, unité virus et immunité, CNRS, URA3015, 28 rue du docteur Roux, F-75015, Paris, France.

${ }^{2}$ Institut Pasteur, département d'immunologie, groupe G5 réponse humorale aux pathogènes, CNRS URA1961, 28, rue du docteur Roux, F-75015, Paris, France. schwartz@pasteur.fr

de criblage et d'isolement d'anticorps monoclonaux humains a révolutionné ce domaine de recherche. Grâce à ces évolutions technologiques, plusieurs dizaines de bNAb « de nouvelle génération », inhibant in vitro, à de très faibles concentrations, près de $90 \%$ des isolats viraux connus sont maintenant disponibles [2].

Les anticorps neutralisants agissent en se fixant à l'enveloppe virale, et ils inhibent l'entrée du virus dans la cellule. La protéine d'enveloppe (Env), présente sous forme de trimères à la surface des virions, est constituée de deux sous-unités : la glycoprotéine gp 120, responsable de la liaison au récepteur CD4, et la glycoprotéine transmembranaire gp4l, qui assure la fusion des membranes. Malgré la capacité du VIH-l à masquer les régions les plus conservées de son enveloppe, par exemple en les protégeant d'un bouclier de glycanes, quatre domaines de l'enveloppe représentent les cibles privilégiées des bNAb : le site de liaison à CD4 (CD4bs, CD4 binding site), les boucles variables V1/V2 et V3 associées aux glycanes, ainsi que la région proximale externe de la sous-unité gp4l (MPER) [2]. 